\title{
Studies of Energy-Linked Reactions
}

\section{NET SYNTHESIS OF ADENOSINE TRIPHOSPHATE BY ISOLATED ADENOSINE TRIPHOSPHATE SYNTHASE PREPARATIONS: A ROLE FOR LIPOIC ACID AND UNSATURATED FATTY ACIDS}

\author{
By DAVID E. GRIFFITHS \\ Department of Molecular Sciences, University of Warwick, Coventry CV4 7AL, U.K.
}

(Received 13 September 1976)

\begin{abstract}
ATP synthase preparations [Complex V, proton-translocating ATPase (adenosine triphosphatase) and oligomycin-sensitive ATPase] contain stoicheiometric amounts of lipoic acid residues (up to $6 \mathrm{~mol}$ of lipoic acid/mol of ATPase complex) and catalyse net ATP synthesis in an uncoupler-and oligomycin-sensitive reaction utilizing dihydrolipoate, oleoyl-CoA and oleic acid, or in a reaction utilizing oleoyl-S-lipoate. The terminal reactions of oxidative phosphorylation are thus analogous to those of substrate-level phosphorylation.
\end{abstract}

The ATP synthase complex, a component of the systems concerned in oxidative phosphorylation, has been isolated in various states of functional integrity from yeast (Tzagoloff \& Meagher, 1971), heart mitochondria (Hatefi et al., 1974; Serrano et al., 1976) and bacterial systems (Sone et al., 1975). It contains the $F_{1}$-ATPase* (five subunit proteins), oligomycin-sensitivity-conferring protein and two to six additional subunits (or membrane sector), which contain the binding sites for oligomycin, triethyltin and $N N^{\prime}$-dicyclohexylcarbodi-imide. Genetic studies of inhibitor resistance (Griffiths, 1972; Lancashire \& Griffiths, 1975) in yeast have shown that at least three different cytoplasmic determinants are involved that presumably specify at least three different protein components of the membrane sector. The genetic evidence thus supports the concept that the various inhibitors of the ATP synthase complex react at different loci in a multi-step enzymic reaction sequence.

The present paper demonstrates that the ATP synthase complex from heart mitochondria contains stoicheiometric amounts of lipoic acid, and that this detergent-dispersed complex, in the absence of added phospholipid, catalyses the net synthesis of ATP in a system utilizing dihydrolipoic acid, oleic acid and oleoyl-CoA. The reaction is sensitive to oligomycin, venturicidin, triethyltin, $N N^{\prime}$-dicyclohexylcarbodi-imide and to uncoupling agents. The reaction sequence appears to be analogous to that catalysed by lipoate succinyltransferase and succinyl-CoA synthetase (EC 6.2.1.4) in substrate-level phosphorylation. These observations indicate that the terminal reactions of oxida-

* Abbreviations: ATPase, adenosine triphosphatase; OS-ATPase, oligomycin-sensitive ATPase complex; succinyl-CoA, 3-carboxypropionyl-CoA. tive phosphorylation leading to the synthesis of ATP in mitochondria involve chemical intermediates, and that the reaction steps involved are contained within the isolated ATP synthase complex.

\section{Materials and Methods}

Heart submitochondrial particles (referred to as 'particles') were prepared as described by Beyer (1967) and heart Complex $V$ by the method of Hatefi et al. (1974). Proton-translocating ATPase was prepared as described by Serrano et al. (1976); the '38-45p fraction' described by them was used. The sources of reagents and inhibitors, protein assays, $P_{1}$ and ATPase assays and preparation of yeast OS-ATPase were as described by Griffiths \& Houghton (1974). Solutions of dihydrolipoate were made up in $0.25 \mathrm{M}$-sucrose $/ 20 \mathrm{~mm}$-Tris/ $\mathrm{HCl}$ buffer containing $0.5 \mathrm{~mm}$-EDTA to avoid rapid autoxidation. Dihydrolipoate and total thiol were measured with 5,5'-dithiobis-(2-nitrobenzoic acid) (Ellman, 1959).

Net phosphorylation was determined by disappearance of $P_{1}$ in a glucose/hexokinase trap system as described in the legends to the Tables. ATP formation was also assayed as glucose 6-phosphate formation by using glucose 6-phosphate dehydrogenase (Hohorst, 1962).

Lipoic acid content of particles and ATP synthase preparations was determined by bioassay with a lipoic acid-requiring mutant of Escherichia coli (Herbert \& Guest, 1970). Both the growth assay and the oxygen-uptake assay described by these authors were used. Protein samples were extracted with $6 \mathrm{M}-\mathrm{HCl}$ by stirring at $22^{\circ} \mathrm{C}$ for $18 \mathrm{~h}$ and then neutralized with $6 \mathrm{M}-\mathrm{NaOH}$ before assay. Autoclaving with $3 \mathrm{M}-\mathrm{H}_{2} \mathrm{SO}_{4}$ for $2 \mathrm{~h}$ as recommended by 
Herbert \& Guest (1970) was not used, as it led to the destruction of lipoic acid. Lipoic acid content (lipoic acid conjugate content) of heart particles and ATP synthase preparations was also determined by an independent method by reaction with $\left[{ }^{3} \mathrm{H}\right]$ dibutylchloromethyltin chloride, a reagent which appears to react specifically with lipoic acid residues in the mitochondrial inner membrane. The method involves reaction of particles with excess of $\left[{ }^{3} \mathrm{H}\right]$ dibutylchloromethyltin chloride and washing with suspension buffer four times by centrifugation at $105000 \mathrm{~g}$ before determination of $\left[{ }^{3} \mathrm{H}\right]$ dibutylchloromethyltin content by liquid-scintillation counting. ATP synthase preparations were treated with excess of $\left[{ }^{3} \mathrm{H}\right]$ dibutylchloromethyltin $(30 \mathrm{nmol} / \mathrm{mg}$ of protein) and precipitated and washed with $5 \%$ $(w / v)$ trichloroacetic acid before liquid-scintillation counting.

Oleoyl-S-lipoate was synthesized by reaction, in aq. $50 \%(\mathrm{v} / \mathrm{v})$ tetrahydrofuran at $\mathrm{pH} 7-8$, of equimolar amounts of oleoyl chloride and dihydrolipoate as described by Seubert (1960) for the synthesis of palmitoyl-CoA. The reaction mixture was concentrated to an oil in a rotary evaporator, acidified to $\mathrm{pH} 1$ with $\mathrm{HCl}$, and extracted with chloroform, and partially purified by washing the chloroform solution with $50 \mathrm{~mm}-\mathrm{Tr}$ is $/ \mathrm{HCl}$ buffer, pH 8, to extract oleic acid and lipoic acid. The partially purified product, which contained approx. $70 \%$ of acyl thioester as determined by the hydroxamate method of Snyder \& Stephens (1959), was used as a solution in dimethylformamide. The preparation still contained oleic acid and lipoic acid.

Oleoyl phosphate was synthesized from oleoyl chloride and anhydrous phosphoric acid essentially as described by Hildebrand \& Spector (1969).

\section{Results}

Initial studies of the lipoic acid content of heart particles by bioassay and $\left[{ }^{3} \mathrm{H}\right]$ dibutylchloromethyltin binding showed that washed heart submitochondrial particles contained 7-10 nmol of lipoic acid residues/ $\mathrm{mg}$ of protein. These lipoic acid residues are tightly bound to the mitochondrial inner membrane, but are extractable by chloroform/methanol mixtures and separable from protein and proteolipid components by t.l.c. The major component is a lipophilic acid conjugate that has the properties of an ester of lipoic acid with an unidentified lipid component. Free lipoic acid present is probably a hydrolysis product of this conjugate.

ATP synthase preparations derived from yeast and heart mitochondria contain stoicheiometric amounts (3-6 $\mathrm{mol} / \mathrm{mol}$ of ATP synthase) of lipoic acid or a lipoic acid conjugate, as determined by bioassay and $\left[{ }^{3} \mathrm{H}\right]$ dibutylchloromethyltin binding. Heart ATPase (38-45p fraction), Complex $V$ and yeast

OS-ATPase were shown by bioassay to contain $5-8 \mathrm{nmol}$ of lipoic acid/mg of protein, whereas values of $7-12 \mathrm{nmol}$ of lipoic acid/mg of protein were obtained by the $\left[{ }^{3} \mathrm{H}\right]$ dibutylchloromethyltin-binding assay.

The presence of lipoic acid residues in the mitochondrial inner membrane and in purified ATP synthase complexes indicated a possible role in oxidative phosphorylation. Investigation of the possible role of lipoic acid showed that heart particles in which the respiratory chain has been blocked by antimycin A and rotenone will catalyse net ATP synthesis with dihydrolipoate as substrate. The reaction is uncoupler- and oligomycin-sensitive, indicating the involvement of the mitochondrial oxidative phosphorylation system (Table 1).

When net ATP synthesis by purified ATP synthase preparations (Complex $\mathrm{V}$ and proton-translocating ATPase) was investigated in the same incubation system, no ATP production was observed, which suggested a deficiency in ATP synthase preparations of a component normally present in the mitochondrial inner membrane necessary for generation of an active intermediate. By analogy with substratelevel phosphorylation, where this component is succinate, a requirement for a lipophilic carboxylic acid was indicated. This requirement was met by unsaturated fatty acids, oleate being more effective than linolenate and arachidonate, whereas linoleate was relatively inactive.

Table 2 demonstrates net synthesis of ATP with dihydrolipoate as substrate by the Complex V preparation of Hatefi et al. (1974), and demonstrates a requirement for oleic acid and oleoyl-CoA. With some preparations oleoyl-CoA alone is effective, and this suggests that different preparations

Table 1. Net synthesis of ATP by heart particles with dihydrolipoate

A glucose/hexokinase trap system was used. Heart particles $(2 \mathrm{mg})$, inhibited with antimycin A $(2 \mu \mathrm{g})$ and rotenone $(2 \mu \mathrm{g})$, were incubated aerobically by shaking with $250 \mathrm{mM}$-sucrose $/ 22 \mathrm{mM}$-glucose $/ 2 \mathrm{mM}$ - $\mathrm{MgCl}_{2} /$ $0.5 \mathrm{~mm}$-EDTA/2mM-ADP/6.6mM-phosphate $/ 20 \mathrm{~mm}$-Tris/ $\mathrm{HCl}$ buffer, $\mathrm{pH} 7.3$ /hexokinase (30 units). Dihydrolipoate $(2.85 \mu \mathrm{mol})$ was added to start the reaction. The total volume was $1.5 \mathrm{ml}$, and the temperature $30^{\circ} \mathrm{C}$. Samples were taken for determination of oxidation of dihydrolipoate and uptake of $\mathbf{P}_{\mathbf{i}}$.

\section{Additions}

None

$\mathrm{KCN}(100 \mathrm{nmol})$

Oligomycin $(2 \mu \mathrm{g})$

'Compound 1799' (5 $\mu \mathrm{g})$

$\begin{array}{cc}\begin{array}{c}\text { Dihydrolipoate } \\ \text { oxidized } \\ (\mu \mathrm{mol} / 20 \mathrm{~min})\end{array} & \begin{array}{c}\mathbf{P}_{\mathbf{1}} \text { uptake } \\ (\mu \mathrm{mol} / 20 \mathrm{~min})\end{array} \\ 1.28 & 3.8 \\ 0.05 & 0.2 \\ 0.2 & 0.15 \\ 0.15 & 0.0\end{array}$


Table 2. Net synthesis of ATP by ATP synthase

The glucose/hexokinase trap system was as described in the legend to Table 1. Expt. (A): The Complex V preparation ( $0.5 \mathrm{mg}$ of protein) of Hatefi et al. (1974) was used, and the reaction was started by the addition of $2.85 \mu \mathrm{mol}$ of dihydrolipoate. The following additions were made: oleoyl-CoA $(10 \mathrm{nmol})$, oleate $(10 \mathrm{nmol})$, palmitate $(20 \mathrm{nmol})$ and palmitoyl-CoA $(10 \mathrm{nmol})$. In Expt. (B) the glucose/hexokinase trap system and amount of Complex V were the same, except that the concentration of ADP was $1 \mathrm{~mm}$. The reaction was started by the addition of oleoyl-S-lipoate in dimethylformamide $(10 \mu \mathrm{l})$ instead of dihydrolipoate. Samples were taken for determination of $P_{i}$, glucose 6-phosphate and thiol content.

\section{Additions}

$P_{1}$ uptake

Expt. (A) (dihydrolipoate-driven)

1. Oleoyl-CoA+oleate ( $\mu \mathrm{mol} / 20 \mathrm{~min})$

2. Oleoyl-CoA+oleate+oligomycin $(2 \mu \mathrm{g})$

3. Oleoyl-CoA + 'compound 1799' $(2 \mu \mathrm{g})$

0.1

4. Oleoyl-CoA+palmitate

5. Palmitoyl-CoA+oleate

0.2

0.0

0.0

Expt. (B) (oleoyl-S-lipoate-driven)

1. Oleoyl-S-lipoate $(0.7 \mu \mathrm{mol})$

2. Oleoyl-S-lipoate $(1.4 \mu \mathrm{mol})$

3. As Expt. (B1)+oligomycin $(2 \mu \mathrm{g})$

2.62

4. As Expt. (B1) + 'compound 1799' (5 $\mu \mathrm{g})$

0.05

5. As Expt. (B1)+efrapeptin ( $2 \mu \mathrm{g})$

0.0

$\mathbf{0 . 0}$

of ATP synthase contain variable amounts of unsaturated fatty acid which in some preparations satisfies the requirement for the unsaturated fatty acid.

A specific requirement for unsaturated fatty acids is indicated, and saturated fatty acids and equivalent acyl-CoA derivatives are inhibitory. The function of oleoyl-CoA may be to generate an enzyme-bound oleoyl intermediate or to generate, enzymically or non-enzymically, oleoyl-S-lipoate, a possible intermediate.

The data in Table 2 indicate that the terminal reactions of oxidative phosphorylation involve transacylation and phosphorolysis reactions analogous to those involved in substrate-level phosphorylation. A possible intermediate, oleoyl- $S$-lipoate, was synthesized by reaction of equimolar amounts of oleoyl chloride and dihydrolipoic acid, and the partially purified product was shown to catalyse ATP synthesis with submitochondrial particles and with Complex V (Table 2). Similar results are obtained with the proton-translocating ATPase of Serrano et al. (1976).

Incubation with oleoyl-S-lipoate led to stoicheiometric formation of ATP in the absence of added oleoyl-CoA, in an uncoupler- and oligomycinsensitive reaction. An unexplained finding is that approx. 1.5-2 mol of ATP is produced per mol of
Table 3. ATP synthesis from oleoyl phosphate by Complex $V$

The reaction mixture contained $250 \mathrm{~mm}$-sucrose, $2 \mathrm{~mm}$ $\mathrm{MgCl}_{2}, 0.5 \mathrm{~mm}$-EDTA, $12 \mathrm{~mm}$-glucose, $0.2 \mathrm{~mm}$-ADP, $20 \mathrm{~mm}$-Tris/HCl buffer, $\mathrm{pH} 7.3$, hexokinase (20units) and Complex V $(0.5 \mathrm{mg}$ of protein). The total volume was $1.0 \mathrm{ml}$. The reaction was started by the addition of oleoyl phosphate in dimethylformamide. The reaction was stopped with $\mathrm{HClO}_{4}$, and the glucose 6-phosphate produced was determined by the method of Hohorst (1962)

\begin{tabular}{|c|c|c|}
\hline $\begin{array}{c}\text { Expt. } \\
\text { no. }\end{array}$ & Additions & $\begin{array}{c}\text { Glucose } \\
6 \text {-phosphate } \\
\text { formed } \\
(\mu \mathrm{mol} / 20 \mathrm{~min})\end{array}$ \\
\hline 1 & Oleoyl phosphate $(0.5 \mu \mathrm{mol})$ & 0.375 \\
\hline 2 & Oleoyl phosphate $(1.0 \mu \mathrm{mol})$ & 0.78 \\
\hline 3 & As Expt. $1+$ 'compound 1799' $(5 \mu \mathrm{g})$ & 0.385 \\
\hline 4 & As Expt. $1+$ oligomycin $(2 \mu \mathrm{g})$ & 0.36 \\
\hline 5 & $\begin{array}{l}\text { As Expt. } 1+N N^{\prime} \text {-dicyclohexyl- } \\
\text { carbodi-imide }(2 \mu \mathrm{g})\end{array}$ & 0.0 \\
\hline 6 & As Expt. $1+$ efrapeptin $(2 \mu \mathrm{g})$ & 0.0 \\
\hline
\end{tabular}

oleoyl- $S$-lipoate. As a net increase in dihydrolipoate concentration was not observed in the reaction mixture, it is assumed that further reactions utilizing dihydrolipoate are involved.

ATP synthase was also shown to catalyse net synthesis of ATP from oleoyl phosphate, a possible intermediate analogous to succinyl phosphate in the succinic thiokinase reaction (Nishimura \& Grinnell, 1972). The transphosphorylation reaction is insensitive to 'compound 1799' and to oligomycin, but is sensitive to $N N^{\prime}$-dicyclohexylcarbodi-imide and to efrapeptin, an $F_{1}$-ATPase inhibitor (Table 3).

\section{Discussion}

The demonstration that the mitochondrial inner membrane contains large amounts of a lipoic acidcontaining component that is also a component of the ATP synthase complex indicates a functional role for lipoic acid in the mechanism of oxidative phosphorylation. The data in Table 2 support this conclusion and demonstrate for the first time net synthesis of ATP by an isolated ATP synthase preparation, and indicate that the terminal reactions of oxidative phosphorylation involve chemical intermediates and reaction steps analogous to those involved in substrate-level phosphorylation.

The demonstration that unsaturated fatty acids are specific cofactors of the terminal reactions of oxidative phosphorylation provides an explanation for the well-established association of decreased efficiency of oxidative phosphorylation with deficiency of unsaturated fatty acid and the inability of unsaturated-fatty acid-requiring mutants of $E$. coli 
and yeast to grow on oxidizable substrates (Resnick \& Mortimer, 1966; Silbert \& Vagelos, 1967; Haslam et al., 1971). The demonstration (Table 2) that oleoyl-S-lipoate can act as a substrate for ATP synthase provides evidence in support of an enzymebound oleoyl- $S$-lipoate intermediate in oxidative phosphorylation analogous to succinyl- $S$-lipoate in substrate-level phosphorylation. As oleoyl-Slipoate generates ATP in the absence of added CoA, the ATP synthase probably contains a tightly bound species of CoA, and preliminary studies indicate that one of the subunits of the ATP synthase contains bound pantothenate (D. E. Griffiths \& M. Partis, unpublished work). Further evidence that the ATP synthase reaction mechanism involves a series of intermediate reaction steps comes from the demonstration (Table 3) that ATP synthase will generate ATP from oleoyl phosphate in an $N N^{\prime}$-dicyclohexylcarbodi-imide-sensitive reaction. This reaction is analogous to the synthesis of ATP from succinyl phosphate, a postulated intermediate in the succinic thiokinase reaction (Nishimura \& Grinnell, 1972), and emphasizes again the similarity to the mechanism of substrate-level phosphorylation.

The results described in the present paper indicate that the terminal reactions of oxidative phosphorylation involve a series of reaction steps and enzyme-bound intermediates, involving transacylation, phosphorolysis and transphosphorylation reactions analogous to substrate-level phosphorylation. A lipoic acid conjugate and unsaturated fatty acids (oleic acid), which are contained within the isolated detergent-dispersed ATPase synthase complex, appear to be essential cofactors. These findings now make possible the definition of intermediate steps in the reaction mechanism of oxidative phosphorylation and the sites of action of oligomycin, triethyltin, $N N^{\prime}$-dicyclohexylcarbodi-imide and uncoupling agents by subfractionation of the ATP synthase complex. A number of new partial reactions such as oleate $\rightleftharpoons$ oleoyl lipoate exchange, $P_{1} \rightleftharpoons$ oleoyl phosphate exchange and ATP $\rightleftharpoons$ oleoyl phosphate ex- change are now available for analysis of this system. In addition, specific inhibitors for lipoic acid function in oxidative phosphorylation are available, namely dibutylchloromethyltin chloride and 8methyl-lipoic acid, which are of use in establishing a role for lipoic acid in oxidative phosphorylation, mitochondrial ATPase and ATP-driven energylinked reactions (D. E. Griffiths, unpublished work).

\section{References}

Beyer, R. E. (1967) Methods Enzymol. 10, 186-194

Ellman, G. L. (1959) Arch. Biochem. Biophys. 82, 70-77

Griffiths, D. E. (1972) in Mitochondria: Biogenesis and Bioenergetics (van der Bergh, S. G., Borst, P. \& Slater, E. C., eds.), pp. 95-104, North-Holland, Amsterdam

Griffiths, D. E. \& Houghton, R. L. (1974) Eur. J. Biochem. 46, 157-167

Haslam, J. M., Proudlock, J. W. \& Linnane, A. W. (1971) J. Bioenerg. 2, 351-370

Hatefi, Y., Stiggall, D. L., Galante, Y. \& Hanstein, W. G. (1974) Biochem. Biophys. Res. Commun. 61, 313-321

Herbert, A. A. \& Guest, J. R. (1970) Methods Enzymol. 18A, 269-272

Hildebrand, J. G. \& Spector, L. B. (1969) J. Biol. Chem. 244, 2606-2613

Hohorst, H. J. (1962) in Methods of Enzymatic Analysis (Bergmeyer, H. U., ed.), pp. 134-138, Verlag Chemie, Weinheim

Lancashire, W. E. \& Griffiths, D. E. (1975) Eur. J. Biochem. 51, 403-413

Nishimura, J. S. \& Grinnell, F. (1972) Adv. Enzymol. 36, 183-202

Resnick, M. A. \& Mortimer, R. K. (1966) J. Bacteriol. 92, 597-600

Serrano, R., Kanner, B. I. \& Racker, E. (1976) J. Biol. Chem. 251, 2453-2461

Seubert, W. (1960) Biochem. Prep. 7, 80-83

Silbert, D. F. \& Vagelos, P. R. (1967) Proc. Natl. Acad. Sci. U.S.A. 58, 1579-1586

Sone, N., Yoshida, M., Hirata, H. \& Kagawa, Y. (1975) J. Biol. Chem. 250, 7917-7923

Snyder, F. \& Stephens, N. (1959) Biochim. Biophys. Acta 34, 244-245

Tzagoloff, A. \& Meagher, P. (1971) J. Biol. Chem. 248, 7328-7338 